\title{
A Computational Method to Calculate the Longitudinal Wave Evolution Caused by Interfaces Between Isotropic Media
}

\author{
Flávio Buiochi, Oscar Martínez, Luis Gómez-Ullate, and Francisco Montero de Espinosa, Member, IEEE
}

\begin{abstract}
This paper presents a computational method to calculate the reflected and transmitted ultrasonic fields at interfaces of complex geometry. The method is performed in two steps. As first step, the velocity potential impulse response from an arbitrary aperture is determined at the interface using the Rayleigh integral and considering the reflection and transmission coefficients. In a second step, the simulated fields are calculated by applying the RayleighSommerfeld integral to the whole, extended interface. In order to validate the method, some experimental cases as, for instance, plane and cylindrical concave surfaces between two media (water-acrylic) were tested. The experimental ultrasonic fields are in good agreement with those provided by the model. Furthermore, in the work, the compromise between the accuracy of the method and the computation time is studied.
\end{abstract}

\section{INTRODUCTION}

$\mathrm{T}$ HE investigation of the acoustic field generated by a broadband ultrasonic transducer through interfaces is an important step for nondestructive evaluation of structures using ultrasound because, in most of the cases, liquid or solid wedges of diverse geometry are used between the transducer face and the structure. Moreover, acoustic beam distortion due to the presence of changes of materials during the propagation, with all kind of geometries, distorts the wavefront. The ultrasonic field depends on the geometry of the transducer, the coupling material (liquids, solid-focusing lenses, and solid wedges), and the incident angle at the interfaces. Theoretical study and field simulations are needed to interpret correctly the echographic data obtained by the transmit-receive response from defects.

Different methods have been developed to study the spatial-temporal characteristics of acoustic fields radiated by broadband transducers. One of these methods uses the spatial impulse response to determine the time-dependent pressure at a spatial point [1]. The starting point is the Rayleigh integral based on Huygens' principle, from which each point of a traveling wavefront can be considered as a

Manuscript received December 23, 2002; accepted September 11, 2003.

F. Buiochi is with the Escola Politécnica da Universidade de São Paulo, Dept. of Mechatronics Engineering, São Paulo, SP, Brazil (email: fbuiochi@usp.br).

O. Martínez and L. Gómez-Ullate are with the Instituto de Automática Industrial-CSIC, Systems Departament, Madrid, Spain.

F. Montero de Espinosa is with the Instituto de Acústica-CSIC, Signals, Systems, and Ultrasonic Technologies, Madrid, Spain. secondary source of hemispherical disturbance. The acoustic field results from the superposition of hemispherical waves radiated by infinitesimal areas from the transducer. This method, based on the Rayleigh integral, also is used for calculation of pressure fields through interfaces $[2]-[6]$. Finite difference methods also have been used for the simulation of acoustic propagation through interfaces [7], [8]. Another approach to study the interaction with interfaces is the conjugate gradient Rayleigh method, primarily developed to study the interaction of ultrasonic waves with rough interfaces [9], [10]. The ray-tracing technique, being faster than the other methods, is not accurate to show the wave distortion at the interfaces [11], [12].

In this paper a three-dimensional computational method, which is based on the spatial impulse response [1] and on the discrete representation computational concept [13], [14], is proposed to calculate the reflected and transmitted ultrasonic fields with interfaces of complex geometry [15]. Both the transducer aperture and the interface are considered as a finite number of elementary sources, each emitting a hemispherical wave. The method is valid for all field regions and may be performed for any excitation waveform radiated from an arbitrary acoustic aperture. Furthermore, it satisfies naturally the Snell's law to evaluate the reflection and refraction angles. The number of elementary areas used to discretize the aperture and the interface limits the precision of the computational method. The accuracy of the approach depends on an adequate choice of the temporal sampling.

To illustrate the usefulness of the computational method to determine the acoustic field evolution through interfaces, the radiation from a circular piston was considered in this work. In order to check the model, a virtual interface placed in front of the circular piston was used to calculate the transmitted field comparing it with the exact solution. In addition, experimental measurements were performed to compare them with the simulated data.

The exact solution of a circular piston mounted within an infinite baffle radiating into a medium is well-known and will not be described here [16]-[20]. The computational method is described for an arbitrary aperture in Section II. Section III presents the comparison between the exact and the presented model solutions and analyzes the main computational parameters (the temporal and spatial sampling). In Section IV, experimental results using several interfaces are compared with the computed data. The conclusions are presented in Section V. In Appendix A, it 


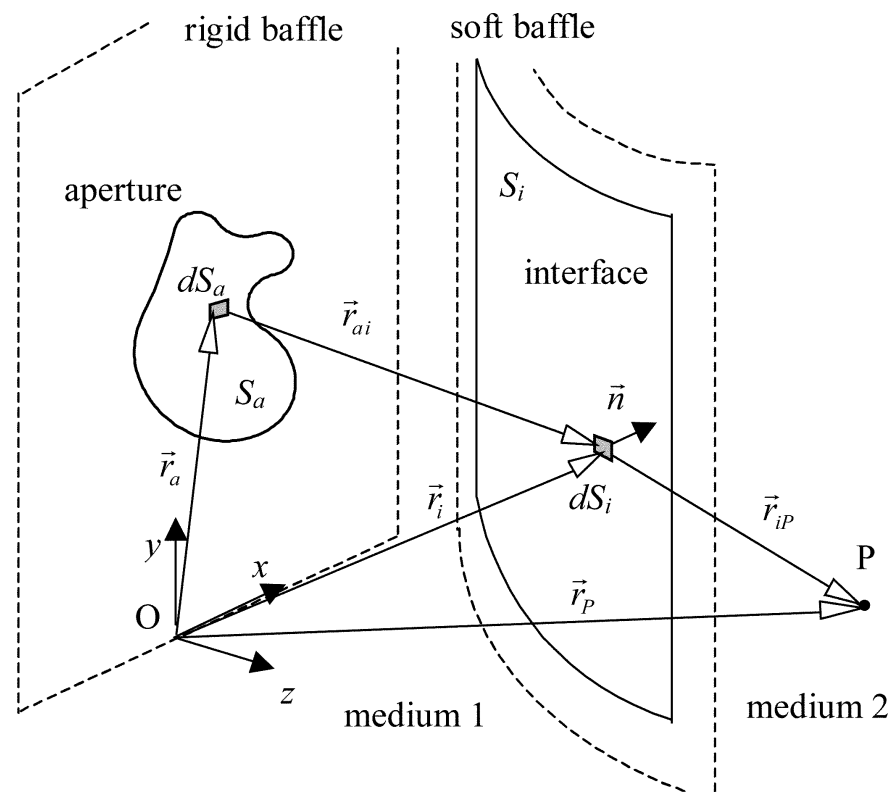

Fig. 1. Geometry used to calculate the field produced by an arbitrary source through an arbitrary interface.

is proven that the theoretical development of the computational method can be extended to a general solution considering three cases of boundary conditions. In Appendix $\mathrm{B}$, the discrete representation computational concept introduced by Piwakowski and Delannoy [13] is described.

\section{THEORY}

Consider an aperture with an arbitrary surface $S_{a}$ embedded in an infinite rigid baffle that is in contact with a medium 1 (Fig. 1). The acoustic pressure field at a point of an isotropic medium can be calculated in the time domain from the Rayleigh integral [1]:

$$
p\left(\vec{r}_{i}, t\right)=\frac{\rho_{1}}{2 \pi} \frac{\partial}{\partial t} \int_{S_{a}} \frac{v_{n}\left(\vec{r}_{a}, t-r_{a i} / c_{1}\right)}{r_{a i}} d S_{a},
$$

where $c_{1}$ is the acoustic propagation velocity in medium $1, \rho_{1}$ is the density of medium $1, r_{a i}$ is the distance from the elementary area $d S_{a}$ located at $\vec{r}_{a}$ to the point located at $\vec{r}_{i}, S_{a}$ indicates the surface of the radiating aperture, and $v_{n}\left(\vec{r}_{a}, t\right)$ is the normal velocity in each point of the aperture.

The interface between media 1 and 2 is approximated to a finite interface, large enough to intercept the major portion of the significant incident energy of the acoustic field. After using (1) to calculate the incident acoustic pressure in the entire interface before the interaction with it, the reflected and transmitted pressure wave $p^{R / T}\left(\vec{r}_{i}, t\right)$ (after the interaction of incident waves with the interface) can be approximated by the following:

$$
p^{R / T}\left(\vec{r}_{i}, t\right)=C^{R / T}\left(\theta_{i}\right) p\left(\vec{r}_{i}, t\right),
$$

where $C^{R / T}\left(\theta_{i}\right)$ is referred to as the wave reflection and transmission coefficients at the interface which depend on the angle of incidence of a plane wave incident on each point of the interface (the plane wave is an approximation of the wavefront generated by the aperture). This dependence is constant for a planar interface; and, in this work, it also is assumed constant for a curved interface, using an average coefficient inside the geometrically illuminated region by the aperture at the interface.

Now, considering that the acoustic pressure immediately after the interaction with the interface is known from (2), it is possible to calculate the reflected and transmitted acoustic fields applying the Rayleigh-Sommerfeld integral to the interface, assuming that it is embedded in an infinite soft baffle [21]:

$$
p\left(\vec{r}_{p}, t\right)=\frac{1}{2 \pi c_{\mathrm{M}}} \int_{S_{i}} \frac{\left|\cos \left(\vec{r}_{i P}, \vec{n}\right)\right|}{r_{i P}} \frac{\partial}{\partial t} p^{R / T}\left(\vec{r}_{i}, t-\frac{r_{i P}}{c_{\mathrm{M}}}\right) d S_{i},
$$

where $c_{\mathrm{M}}$ is the acoustic propagation velocity of medium $\mathrm{M}$, which is 1 for medium 1 (when reflected waves are computed) and 2 for medium 2 (when transmitted waves are computed); $r_{i P}$ is the distance from the elementary area $d S_{i}$ located at $\vec{r}_{i}$ to the field point located at $\vec{r}_{p} ; \cos \left(\vec{r}_{i P}, \vec{n}\right)$ is the cosine of the angle between the normal vector $\vec{n}$ and the vector $\vec{r}_{i P}$, and its modulus guarantees to use the same expression to the cases of reflection and transmission; $S_{i}$ indicates the surface of the interface. The integration over the boundary between the two media is truncated over a finite interface $S_{i}$, and an ideally soft baffle is considered. In this work, we always choose a finite interface that is large enough to intercept the main incident energy of the acoustic beam.

It is well-known that the integrals (1) and (3) are valid for a planar radiator. However, these integrals can approximately represent the radiation of a curved source under certain conditions: the radiating surface is slightly curved, and the source dimension is large compared with the wavelength [22]-[24]. Under these conditions, most of the radiated energy, which is concentrated close to the central beam, will be affected very little by the baffle around the radiating surface [24]. The approach we are using in this work deals with planar apertures and curved interfaces that satisfy the above conditions.

The solution for $p\left(\vec{r}_{p}, t\right)$ presented in (3) can be simplified, assuming that the aperture is a uniform piston. Substituting (1) and (2) into (3) and after some calculations, it follows that the transient field is determined by a temporal convolution between the excitation signal $v(t)$ and the velocity potential impulse response $h\left(\vec{r}_{p}, t\right)$ :

$$
p\left(\vec{r}_{p}, t\right)=\rho_{1} \frac{\partial v(t)}{\partial t} * h\left(\vec{r}_{p}, t\right)
$$

where $*$ indicates the time convolution, and $h\left(\vec{r}_{p}, t\right)$ is the velocity potential impulse response at the field point $\mathrm{P}$, which is defined by:

$$
h\left(\vec{r}_{p}, t\right)=\frac{1}{2 \pi c_{\mathrm{M}}} \int_{S_{i}} \frac{\left|\cos \left(\vec{r}_{i P}, \vec{n}\right)\right|}{r_{i P}} \frac{\partial}{\partial t} h_{a}\left(\vec{r}_{i}, t-\frac{r_{i P}}{c_{\mathrm{M}}}\right) d S_{i},
$$


where $h_{a}\left(\vec{r}_{i}, t\right)$ is the velocity potential impulse response at the interface due to the radiation from the aperture and the wave evolution caused by the interface:

$$
h_{a}\left(\vec{r}_{i}, t\right)=\frac{C^{R / T}\left(\theta_{i}\right)}{2 \pi} \int_{S_{a}} \frac{\delta\left(t-r_{a i} / c_{1}\right)}{r_{a i}} d S_{a} .
$$

A detailed general deduction of (4), (5), and (6) is shown in Appendix A, considering that the infinite baffle around the interface can be one of the three basic types of boundary conditions: rigid, soft, and free-field [25]. This model neglects field components, which appear at a fluid/solid interface, like the converted waves, shear waves, surface waves, head waves, etc.

In this work, computation is divided into two parts, using the approach proposed in [13], [14] valid for an arbitrary aperture (as shown in Appendix B). In a first step, after dividing the aperture into a number of elementary areas, the velocity potential impulse response $h_{a}\left(\vec{r}_{i}, t\right)$ is calculated from (6) at the whole-extended interface, which also has been approximated by a number of elements of small area. In each of the discretized elements of the interface, the impulse response function is affected by the corresponding reflection and/or transmission coefficients. In a second step, the velocity potential impulse response $h\left(\vec{r}_{p}, t\right)$ is calculated from (5), and the reflected and transmitted pressure fields are obtained by applying (4) at every field point $\mathrm{P}$.

The time-averaged discrete impulse response [13], [14] is the computational solution used to calculate the velocity potential impulse responses at the point $\mathrm{P}$ and at the interface, respectively given by (5) and (6). The temporal sampling $\Delta t$ is used constant during the calculation of the impulse responses, but the spatial samplings could be different in the aperture $\left(\Delta x_{a}\right.$ and $\left.\Delta y_{a}\right)$ and in the interface $\left(\Delta x_{i}\right.$ and $\left.\Delta y_{i}\right)$.

\section{Comparison with the Exact Solution}

In this section an implementation of the computational method will be presented for the case of a planar circular piston of radius $9.5 \mathrm{~mm}$ radiating into a medium with a rectangular virtual interface of dimensions $L \times L$ placed in front of the piston (Fig. 2). An angular orientation $\alpha$ and an axial distance $Z$ define the position of the interface relative to the referential $O x z$ located at the center of the piston. All simulations were carried out taking both media 1 and 2 as water to permit a comparison with the exact solution of a piston radiating in water. An excitation signal of $1 \mathrm{MHz}$ sine-wave single cycle was used (wavelength $\lambda=$ $1.5 \mathrm{~mm}$ ). The virtual interface was located at the near field with $Z=15 \mathrm{~mm}$ and $L=25 \mathrm{~mm}$.

Fig. 3 shows a comparison between the exact pressure responses and the corresponding model result at six different field points (Fig. 4). Fig. 3(a) shows three different near-field points $(z=20 \mathrm{~mm})$ at the acoustic axis

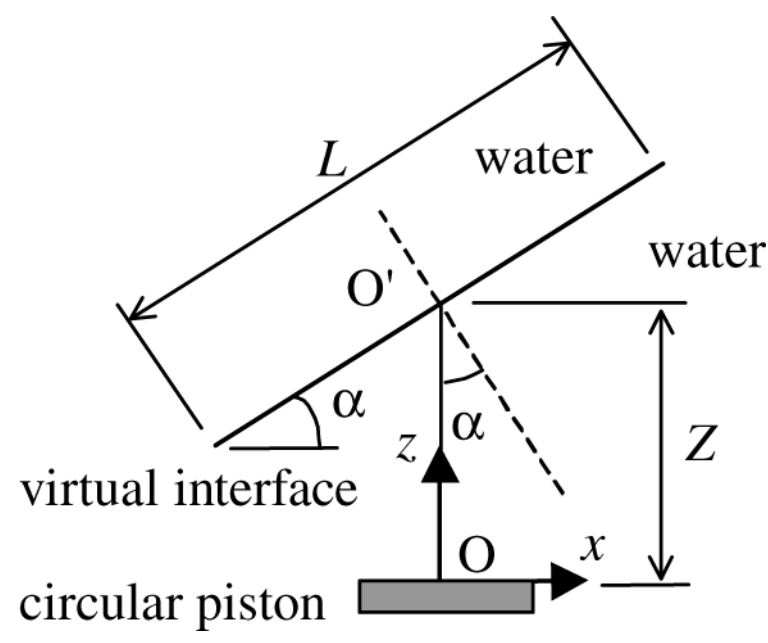

Fig. 2. Illustration of the circular piston radiating into a medium with a virtual interface.

and at 10 and $20 \mathrm{~mm}$ off axis. Fig. 3(b) shows the results at the same $x$ coordinate but at far-field conditions $(z=60 \mathrm{~mm})$. The model responses were calculated with $c \Delta t=\lambda / 15=0.1 \mathrm{~mm}, \Delta x_{a}=\Delta y_{a}=\lambda / 6=0.25 \mathrm{~mm}$, $\Delta x_{i}=\Delta y_{i}=\lambda / 6=0.25 \mathrm{~mm}$, and $\alpha=0^{\circ}$. In all cases the approach gives very good results, except the $P_{3}$ case, which, due to the geometry, is outside the shadow of the interface (Fig. 4). In this point $P_{3}$, the travel time calculated by the computational method $\left(t^{\prime}+t^{\prime \prime}\right)$ is larger than the real one $(t)$. Thus, more accurate results can be obtained if all field points are in the so-called shadow region. Furthermore, we observed that the simulated waveforms include some noise due to the temporal and spatial samplings adopted, and some small spurious signals due to the finite interface used.

To give an indication of the approach accuracy, the error respect to the exact solution of the pressure responses has been calculated for every signal in the time domain as:

$$
e=\sqrt{\frac{1}{N} \sum_{i=1}^{N}\left(s_{E}(i)-s_{C}(i)\right)^{2}}
$$

where $s_{E}(i)$ is the signal computed by the exact solution, $s_{C}(i)$ is the signal computed by our method, and $N$ is the number of samples. To have an idea about the meaning of the error concept used, the errors between the approached pressure responses and the exact solution are displayed in Fig. 3.

Fig. 5 shows the comparison between the exact solution and four different computational cases for two-dimensional simulated fields in the $\mathrm{x}-\mathrm{z}$ plane. All approached fields have been calculated with $c \Delta t=\lambda / 15=0.1 \mathrm{~mm}$ and $\Delta x_{a}=\Delta y_{a}=\lambda / 6=0.25 \mathrm{~mm}$. Cases 1,2 , and 3 were calculated with an angular orientation of the virtual interface $\alpha=0^{\circ}$ using a value of the spatial sampling at the interface $\Delta x_{i}=\Delta y_{i}$ of $\lambda / 6=0.25, \lambda / 3=0.5$, and $\lambda / 1.5=1 \mathrm{~mm}$, respectively. Case 4 was calculated with an angular orientation $\alpha=10^{\circ}$ considering the same computational parameters described in Case 3 . We observed 
(a)
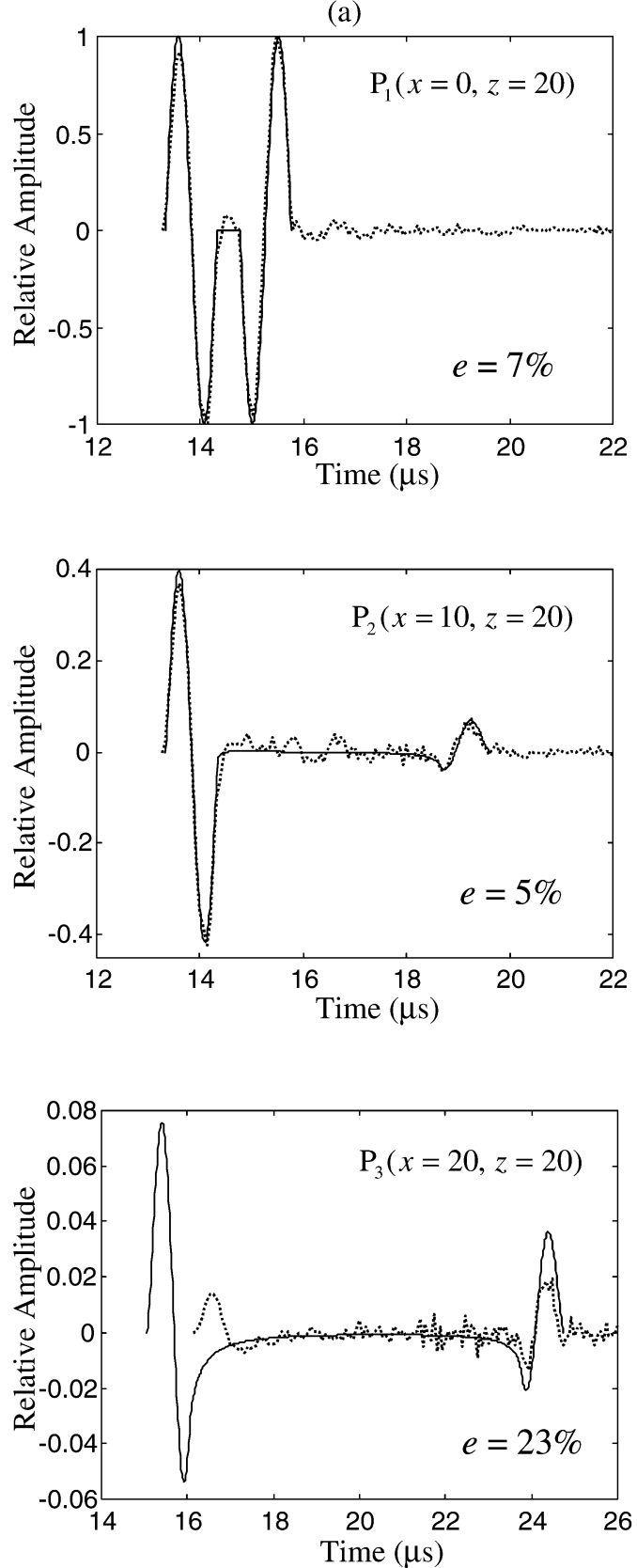

(b)
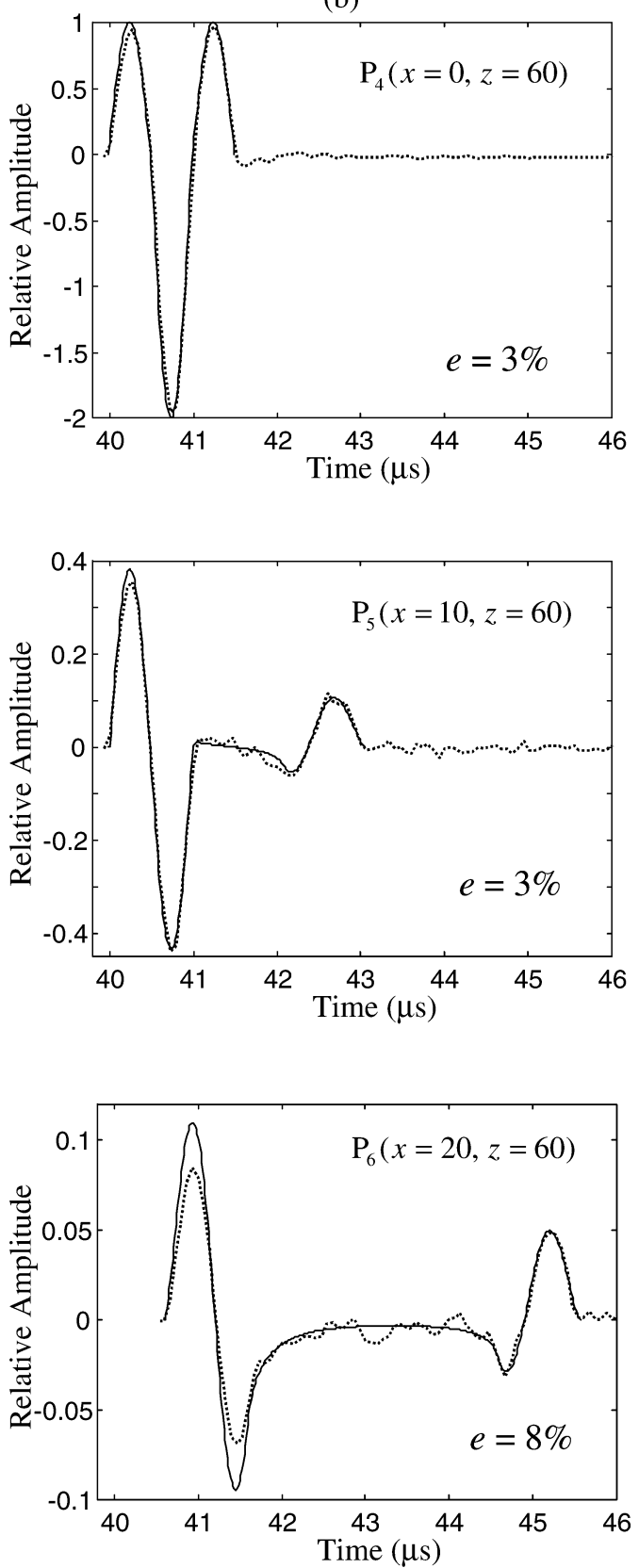

Fig. 3. Comparison of the exact (solid line) and the approached (dotted line) pressure responses in the regions: (a) near field at $z=20 \mathrm{~mm}$ (column on the left), and (b) far field at $z=60 \mathrm{~mm}$ (column on the right).

that the main differences appear in the near-field region and when rough spatial sampling is used. Case 4 shows that the inclined interface decreases the error because it brakes the symmetry of the field calculation. A big difference exists in computation time. For instance, using a 1.4 GHz Pentium IV computer (Intel, Santa Clara, CA), Case 1 takes 25 minutes, and Cases 2, 3, and 4 take only 6 , 1.5, and 1.5 minutes, respectively. The exact solution for the circular piston takes only 4 seconds.

Figs. 6(a) and (b) show the relative errors calculated using (7) for all the points with $|x| \leq 10 \mathrm{~mm}$ at $z=20 \mathrm{~mm}$ and $z=60 \mathrm{~mm}$, for the cases described in Fig. 5. As a general conclusion, the error at near field is always bigger than at far-field conditions, because near-field points are closer to the interface with the worst average of the impulse response that is explained in (18) in Appendix B. To better clarify the effect of the vicinity of the field point to the interface, a comparison of the exact solution with the approached pressure responses calculated at $x=10$, $z=60 \mathrm{~mm}$ placing the interface at two axial distances, $Z=15 \mathrm{~mm}$ and $Z=55 \mathrm{~mm}$, was performed, as shown in Fig. 7. The interface discretization conditions were the same as those used in Case 2 of Fig. 5. The relative errors also are displayed in Fig. 7. The error increases if the point is closer to the interface.

Fig. 8 shows the average relative errors, varying some computational parameters, for two cases: Fig. 8(a) normalized temporal sampling $(c \Delta t)$ for a given spatial sam- 


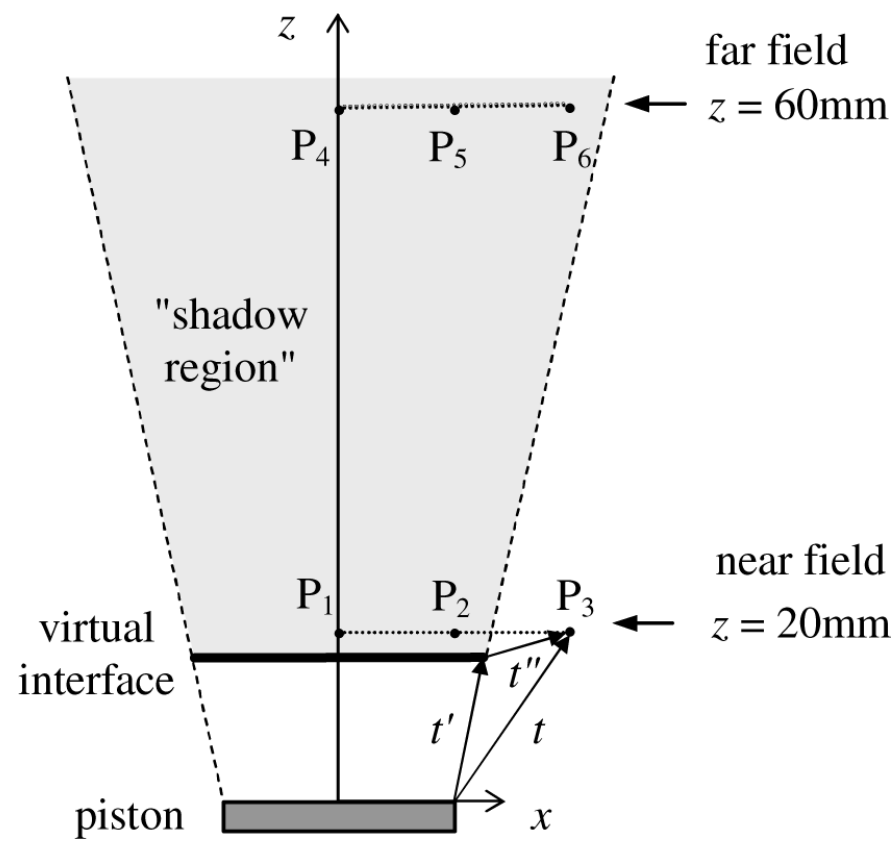

Fig. 4. The shadow region of the virtual interface and the six positions analyzed in Fig. 3.

pling $\left(\Delta_{a, i}=\lambda / 6=0.25 \mathrm{~mm}\right)$, and Fig. 8 (b) dimension of the virtual square interface $(L)$ for two spatial samplings $\left(\Delta_{a, i}=\lambda / 6=0.25 \mathrm{~mm}\right.$ and $\left.\Delta_{a, i}=\lambda / 7.5=0.20 \mathrm{~mm}\right)$. In these simulations, the spatial samplings in the aperture and in the interface are equal to $\Delta_{a, i}$, i.e., $\Delta x_{a}=\Delta y_{a}=$ $\Delta x_{i}=\Delta y_{i}=\Delta_{a, i}$. The average relative errors were calculated using (7) for the range $|x| \leq 10 \mathrm{~mm}$ at $z=20 \mathrm{~mm}$ and $z=60 \mathrm{~mm}$. The interface is placed at $Z=15 \mathrm{~mm}$ with angular orientation of $\alpha=0^{\circ}$. Both Figs. 8(a) and (b) show the computational parameters used for Case 1 described in Fig. 5.

In Fig. 8(a), we note that the election of the optimal temporal sampling is not a key point. The statistical fluctuations are more important when a large temporal sampling is chosen $(c t>\lambda / 10)$. Better resolution is obtained with smaller temporal samplings, but the error is maintained slightly below $5 \%$ with little variations, which is the limit caused by the particular spatial sampling used in the simulations. In relation with the spatial sampling [Fig. 8(b)], the precision of the computational method is proportional to the density of elementary areas in the aperture and in the interface [13], [14]. Furthermore, if the dimensions of the interface are increased, in general less incident energy is lost and the computation errors decrease. As these two aspects increase the number of cells and therefore the computation time, a balance must be found.

\section{Application with Interfaces}

Experiments to measure the acoustic field evolution due to the appearance of interfaces during the propagation have been performed to test the validity of the computational method. All measurements were carried out in water $\left(\rho=1000 \mathrm{~kg} / \mathrm{m}^{3}, c=1500 \mathrm{~m} / \mathrm{s}\right)$ using a $0.2-\mathrm{mm} \operatorname{diam}$
PVDF omnidirectional hydrophone with flat response up to $20 \mathrm{MHz}$ and sensitivity $0.500 \mathrm{pC} /$ bar (VITA 19, Dapco Industries, Inc., Ridgefield, CT). The solid medium used was acrylic (density $\rho=1180 \mathrm{~kg} / \mathrm{m}^{3}$, longitudinal velocity $c_{L}=2700 \mathrm{~m} / \mathrm{s}$, shear velocity $c_{S}=1400 \mathrm{~m} / \mathrm{s}$ ). A $1 \mathrm{MHz} 47 \%$ bandwidth circular transducer with a diameter of $19 \mathrm{~mm}$ (Accuscan series, Panametrics, Waltham, MA) has been used as emitter. The transducer was excited in wideband conditions with a Master Scan 330 (Sonatest Plc Group, Milton Keynes, UK). The reference acoustic signal used into the calculations was measured facing the needle hydrophone very close to the transducer emitting face. A water tank with automatic three-dimensional movements (Microcontrole, Evry, France) and signal acquisition was used. For the sake of simplicity, only planar and cylindrical concave solid surfaces were tested.

\section{A. Planar Surface Case}

For the planar surface case, two physical arrangements were used, which are schematically depicted in Fig. 9. The interface is assumed to have an angular orientation $\alpha$ and is located at an axial distance $Z$ from the center of the circular transducer (point $O$ ). To show the transmitted and reflected acoustic waves, the referential $O^{\prime} \bar{x} \bar{z}$ for the acrylicwater interface [Fig. 9(a)] and the referential $O x z$ for the water-acrylic interface [Fig. 9(b)] have been adopted, respectively.

Fig. 10 shows the $\bar{x} \bar{z}$ scan of the maximum pressure distribution for the experimental and simulated fields refracted at the acrylic-water interface, with an incidence angle $\alpha=45^{\circ}$ and an axial distance $Z=20.7 \mathrm{~mm}$. In Fig. 10, both interface and transducer used in the simulation are represented graphically. The reference system $O^{\prime} \bar{x} \bar{z}$ [see Fig. 9(a)] has been considered to represent the images. The calculated and measured beam profiles at three different distances from the interface $(\bar{z}=4,38$, and $72 \mathrm{~mm})$ are shown in Fig. 11. The pressure amplitude was normalized by the maximum value recorded at $\bar{z}=38 \mathrm{~mm}$.

Fig. 12 refers to another case with $\alpha=15^{\circ}$ and $Z=$ $17 \mathrm{~mm}$. In Fig. 12, the measured and calculated beam profiles at three different coordinates $\bar{z}: 4,40$, and $76 \mathrm{~mm}$ are presented. The pressure amplitude was normalized by the maximum value recorded at $\bar{z}=40 \mathrm{~mm}$.

In all cases, the agreement between the measured and calculated beam profiles is very good.

Fig. 13 shows $x z$ maximum pressure scan concerning the reflected field at the water-acrylic interface, using an incidence angle $\alpha=30^{\circ}$ and an axial distance $Z=30 \mathrm{~mm}$. The coordinate system $O x z$ [see Fig. 9(b)] has been considered. In Fig. 13, both interface and transducer used in the simulation are represented graphically. Fig. 14 shows the measured and calculated beam profiles at $x=16,48$, and $80 \mathrm{~mm}$, which were normalized by the maximum value recorded at $x=48 \mathrm{~mm}$.

To demonstrate the accuracy of the predicted pressure responses of Fig. 13, Fig. 15 shows the reflected measured and simulated waveforms at two points: $P_{1}(x=33.8, z=$ 

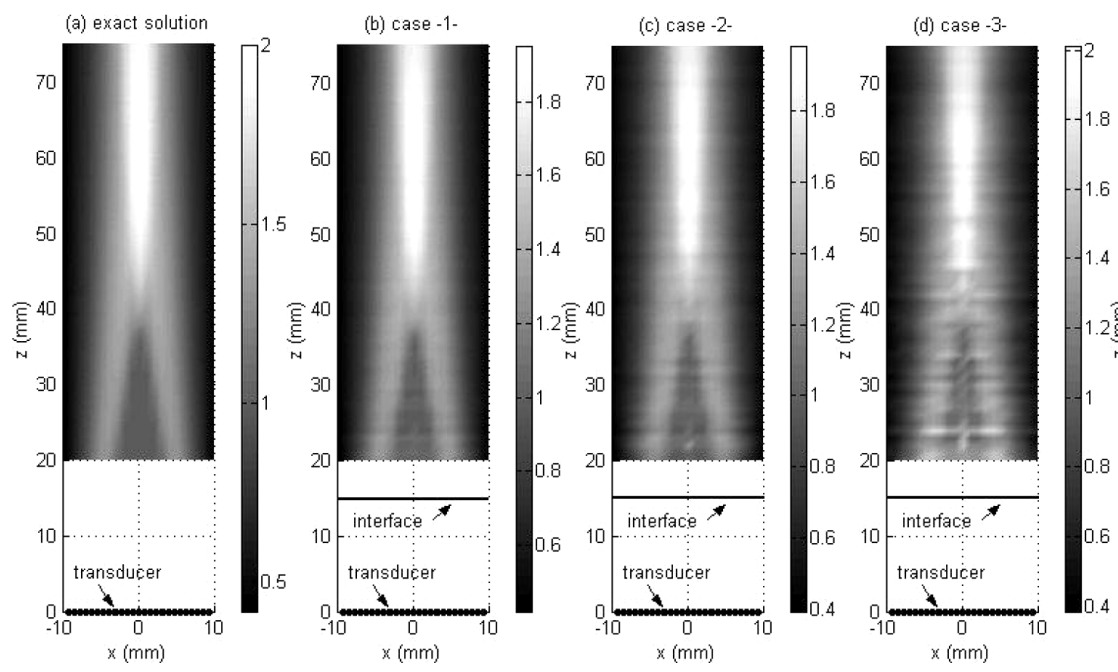

(e) case -4-

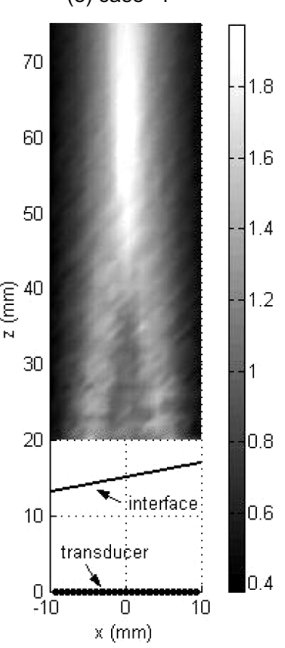

Fig. 5. Comparison of the $x z$ exact (a) and approached maximum pressure scan calculated for four different computational cases: (b) $\Delta x_{i}=$ $\Delta y_{i}=0.25 \mathrm{~mm}$ and $\alpha=0^{\circ}$ (Case 1), (c) $\Delta x_{i}=\Delta y_{i}=0.5 \mathrm{~mm}$ and $\alpha=0^{\circ}$ (Case 2), (d) $\Delta x_{i}=\Delta y_{i}=1 \mathrm{~mm}$ and $\alpha=0^{\circ}($ Case 3 ), and (e) $\Delta x_{i}=\Delta y_{i}=1 \mathrm{~mm}$ and $\alpha=10^{\circ}($ Case 4$)$.

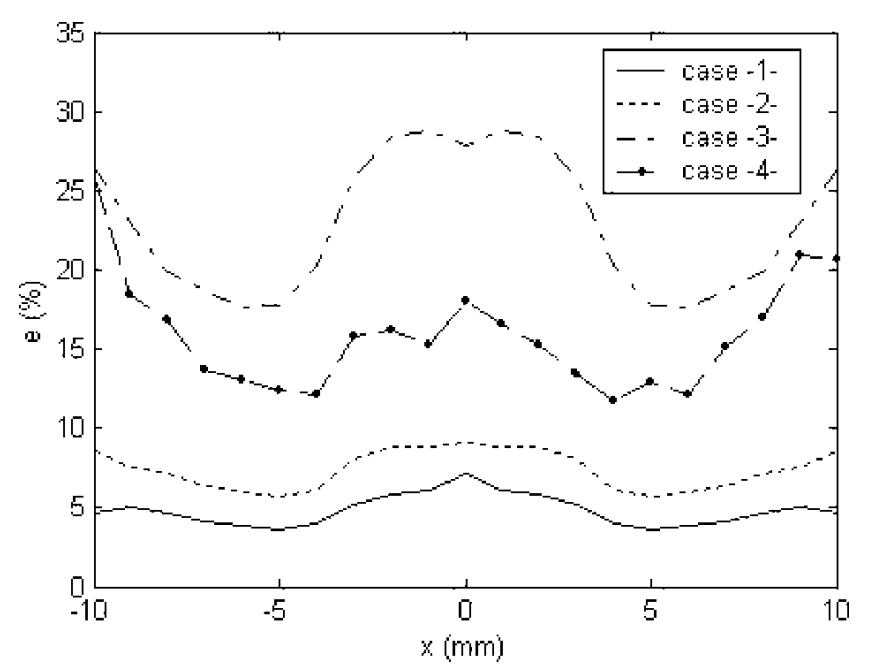

(a)

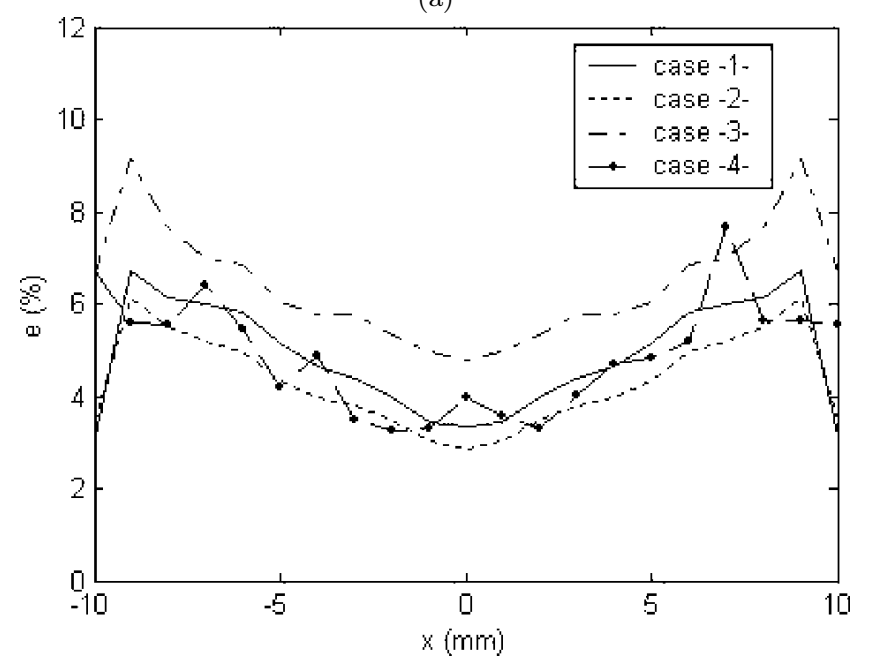

(b)

Fig. 6. The pressure response error $e(\%)$ calculated at (a) $z=20 \mathrm{~mm}$ and (b) $z=60 \mathrm{~mm}$ for the range $|x| \leq 10 \mathrm{~mm}$ for the computational Cases 1-4 described in Fig. 5.
$11.7 \mathrm{~mm})$ and $P_{2}(x=33.8, z=6.7 \mathrm{~mm})$. The amplitudes of the pressure responses were normalized by the maximum value recorded at $P_{1}$. The relative errors are displayed in Fig. 15. As demonstrated previously, the computational method, in fact, accurately predicts the relative amplitude and the time histories of the pressure waveforms.

\section{B. Cylindrical Concave Surface Case}

For the cylindrical concave surface case, two physical arrangements were used, which are illustrated in Fig. 16. The interface is assumed to have an angular orientation $\alpha$ and is located at an axial distance $Z$ from the center of the circular transducer (point $O$ ). The referential $O x z$ has been adopted to show the transmitted and reflected acoustic fields for the acrylic-water [Fig. 16(a)] and for the water-acrylic interfaces [Fig. 16(b)], respectively.

Fig. 17 shows the experimental and the simulated results of the transmitted field through the acrylic-water interface for an angle $\alpha=18^{\circ}$ and an axial distance $Z=19 \mathrm{~mm}$ [Fig. 16(a)]. The beam profiles at three different distances from the transducer $(z=30,70$, and $110 \mathrm{~mm}$ ) are shown in Fig. 18. The pressure amplitude has been normalized by the maximum value recorded at $z=70 \mathrm{~mm}$.

Fig. 19 shows the $x z$ maximum pressure scan of the reflected field at the water-acrylic interface [Fig. 16(b)], using $\alpha=30^{\circ}$ and $Z=30 \mathrm{~mm}$. Fig. 20 shows the beam profiles at four different values of coordinate $x: 16,30,44$, and $58 \mathrm{~mm}$. The pressure amplitude was normalized by the maximum value recorded at $x=30 \mathrm{~mm}$.

From Figs. 17 to 20, it can be observed that the simulated results are in very good agreement with the measured ones, taking into account the difficulty of aligning the transducer, the interface, and the hydrophone in the experimental setup. The only experimental problem comes from the appearance of spurious echoes coming from the 
(a)

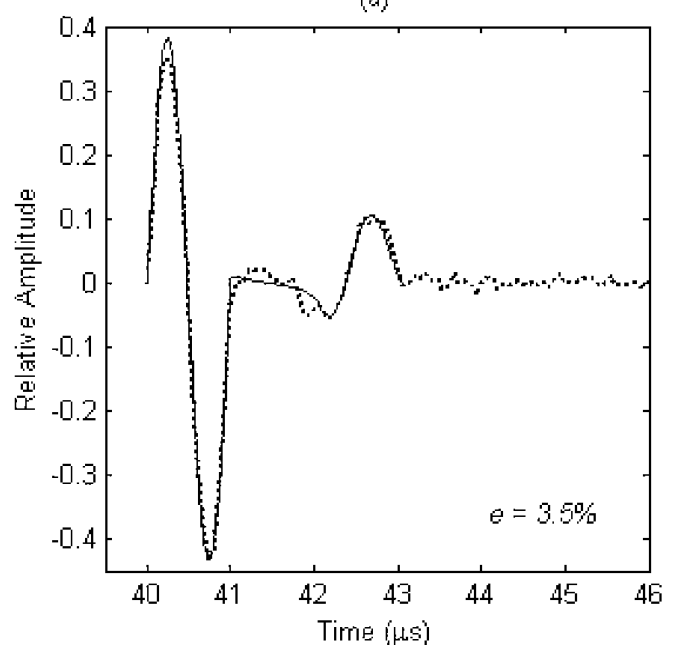

(b)

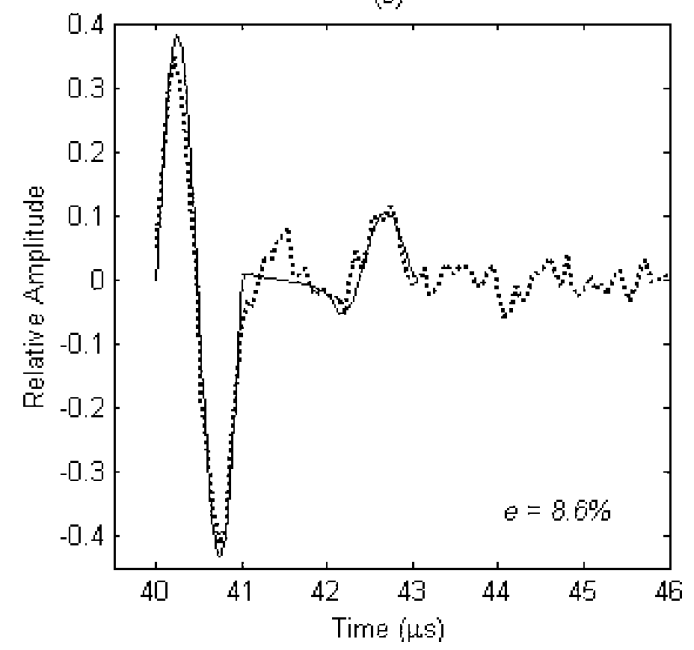

Fig. 7. Comparison of the exact (solid line) and the approached (dotted line) pressures responses at $x=10 \mathrm{~mm}$ and $z=60 \mathrm{~mm}$, placing the interface at two axial distances: (a) $Z=15 \mathrm{~mm}$ (Case 2 Fig. 5) and (b) $Z=55 \mathrm{~mm}$.

(a)

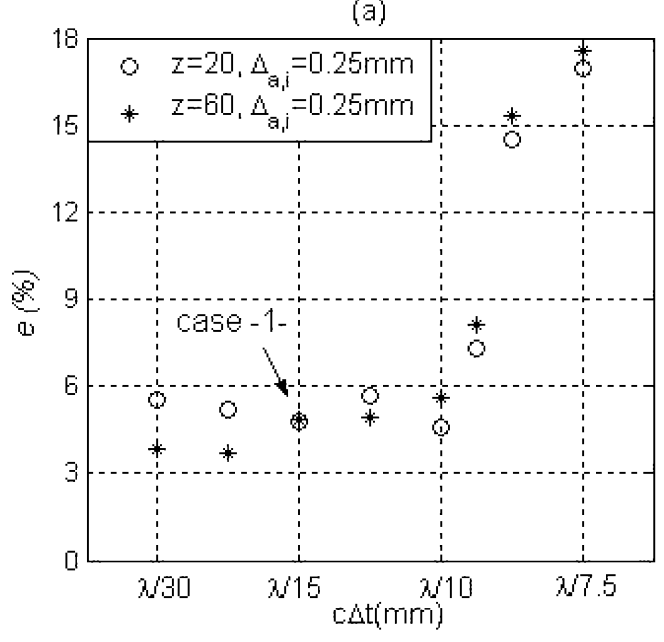

(b)

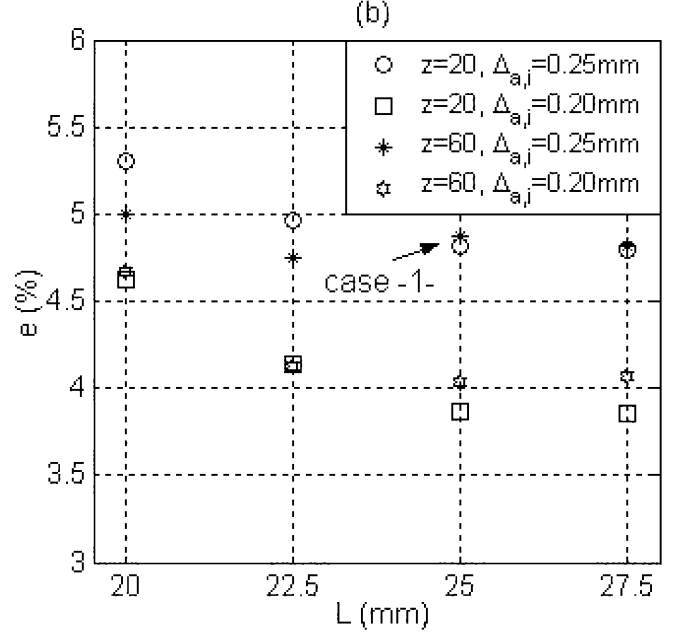

Fig. 8. The average error $e(\%)$ of the pressure responses calculated at $z=20 \mathrm{~mm}$ and $z=60 \mathrm{~mm}$ for the range $|x| \leq 10 \mathrm{~mm}$ as a function of the computational parameters: (a) temporal sampling and (b) dimension of the interface with two spatial samplings.

walls of the acrylic shape used to reflect the signal. These echoes can produce an extra amplitude of the recorded signal (see Figs. 19 and 20) because the analogic window used to detect the echoes when performing the scan must be wide enough to avoid missing the first reflected signal.

\section{Conclusions}

Based on the impulse response and the discrete representation methods, a three-dimensional computational method has been developed to calculate the reflected and transmitted ultrasonic fields through interfaces of complex geometry. The ultrasonic field is calculated by the method of the Rayleigh integral, extended to take into account the wave mode conversion at the interface. A closed-form analytical expression of the velocity potential impulse response has been obtained to describe the reflection and the transmission at the interface. The number of elementary
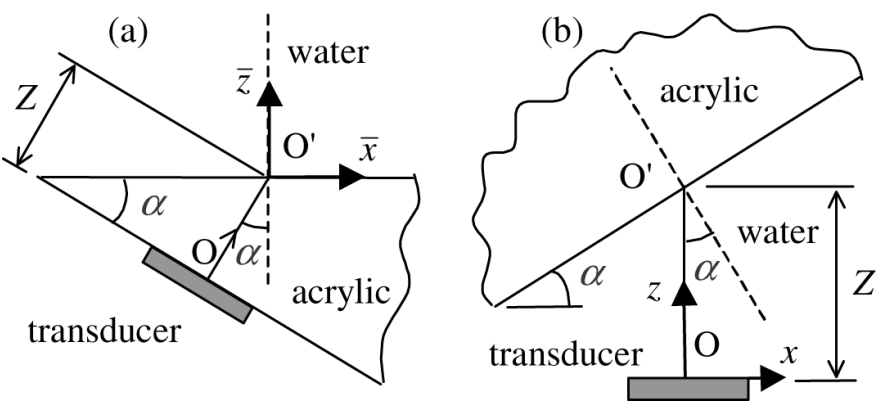

Fig. 9. The geometry of the planar interface cases studied: (a) acrylicwater interface (transmission) and (b) water-acrylic interface (reflection).

areas used to divide the aperture and the interface limits the precision of the computational method. A virtual interface (water-water interface) was used to compare the method with the exact solution for the case of a piston vibrating in water. The difference between the exact solution 

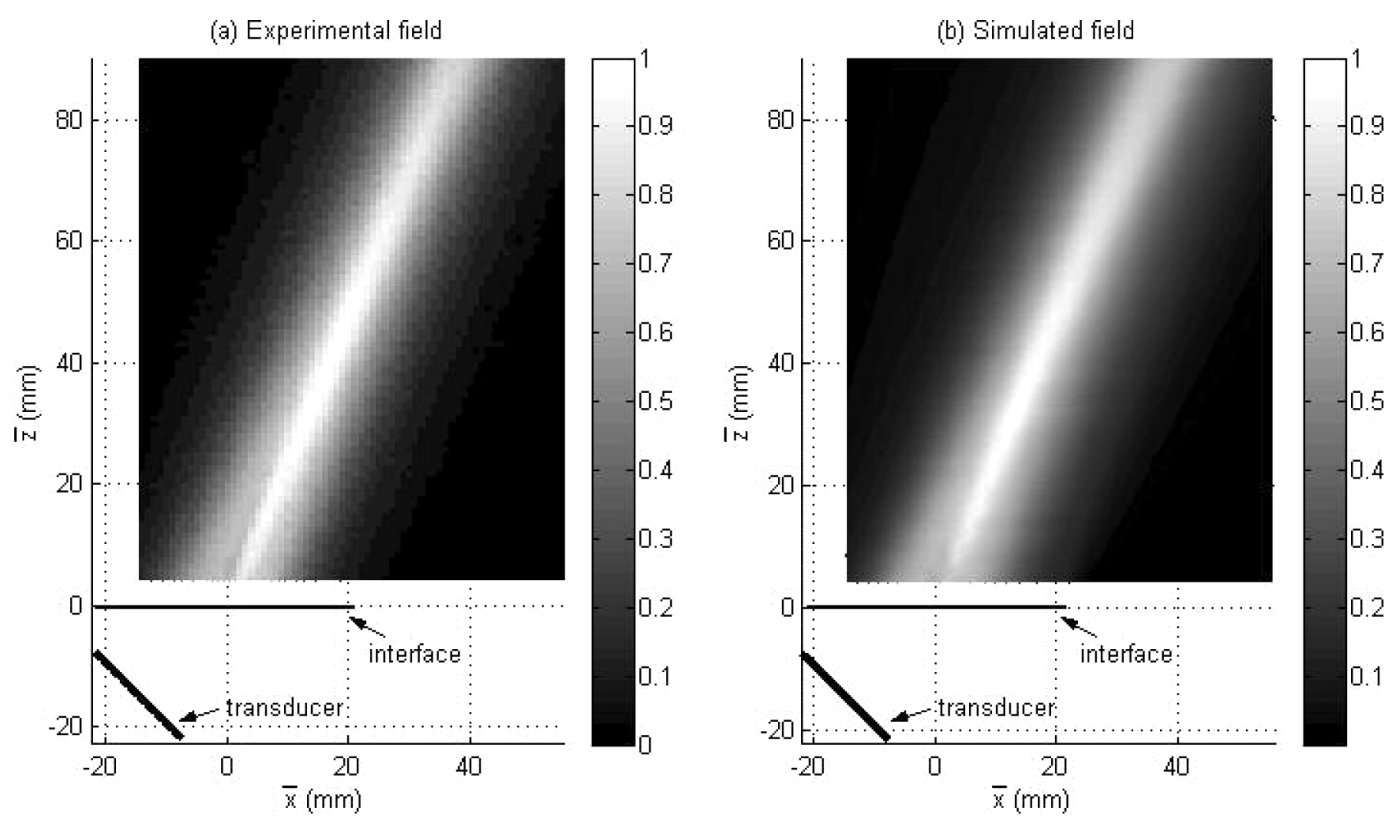

Fig. 10. The experimental (a) and the simulated (b) transmitted fields for $\alpha=45^{\circ}$ for acrylic-water interface.

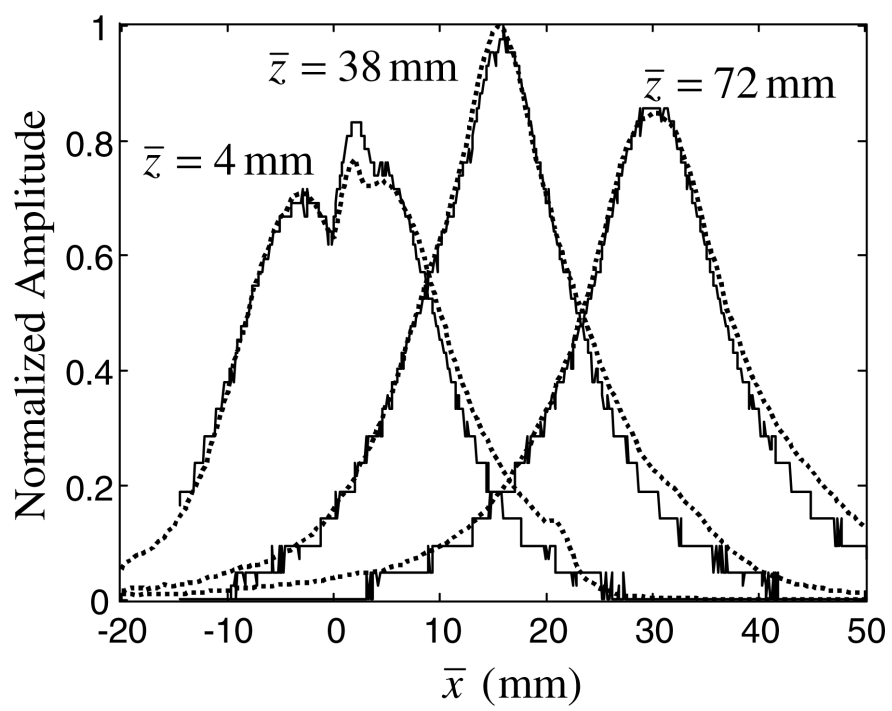

Fig. 11. Comparison of the experimental (solid line) and the simulated (dotted line) data for the transmitted field from the acrylicwater interface with $\alpha=45^{\circ}$ at $\bar{z}=4,38$, and $72 \mathrm{~mm}$.

and the proposed method can be minimized by means of an adequate choice of the temporal sampling. This method can be easily extended for the case of array apertures.

\section{APPENDIX A}

\section{General Deduction}

Under the assumption that the time and space variables are separable at the aperture surface, the normal velocity is given by:

$$
v_{n}\left(\vec{r}_{a}, t\right)=A\left(\vec{r}_{a}\right) v(t),
$$

where $A\left(\vec{r}_{a}\right)$ is the vibration amplitude distribution, and $v(t)$ is the particle velocity function at the aperture (exci-

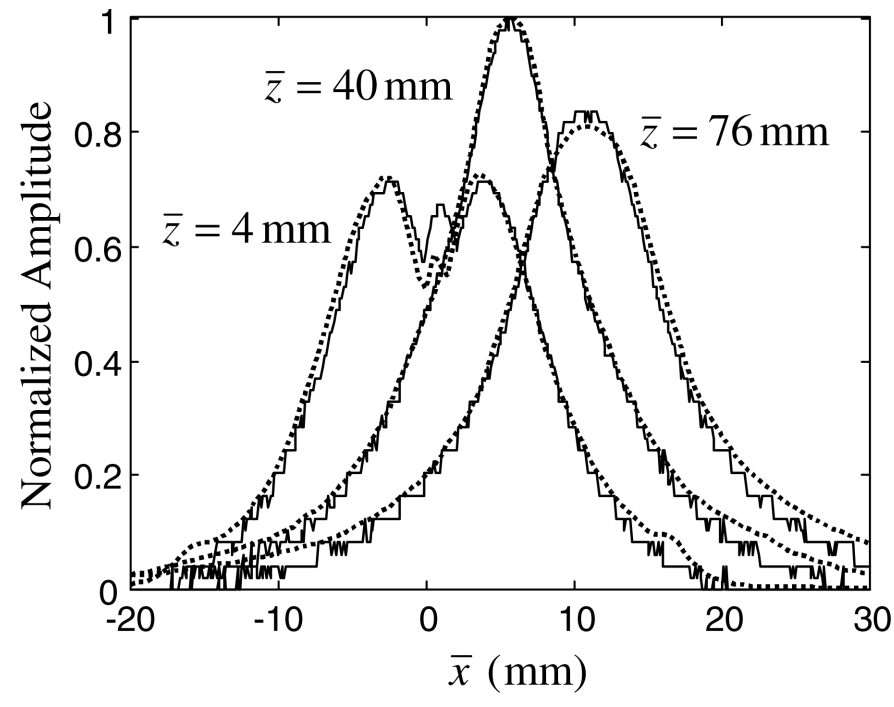

Fig. 12. Comparison of the experimental (solid line) and the simulated (dotted line) beam profiles of the transmitted field from the acrylic-water interface with $\alpha=15^{\circ}$ at $\bar{z}=4,40$, and $76 \mathrm{~mm}$.

tation signal). Thus, the term $v_{n}\left(\vec{r}_{a}, t-r_{a i} / c_{1}\right)$ in (1) can be written as:

$$
v_{n}\left(\vec{r}_{a}, t-\frac{r_{a i}}{c_{1}}\right)=A\left(\vec{r}_{a}\right) \int_{-\infty}^{+\infty} v(\tau) \delta\left(t-\frac{r_{a i}}{c_{1}}-\tau\right) d \tau .
$$

If (1) and (9) are inserted into (2) and the order of integration is inverted, the result is:

$$
\begin{aligned}
& p^{R / T}\left(\vec{r}_{i}, t\right)=\rho_{1} \frac{\partial}{\partial t} \int_{-\infty}^{\infty} v(\tau) \\
& {\left[\frac{C^{R / T}\left(\theta_{i}\right)}{2 \pi} \int_{S_{a}} \frac{A\left(\vec{r}_{a}\right)}{r_{a i}} \delta\left(t-\frac{r_{a i}}{c_{1}}-\tau\right) d S_{a}\right] d \tau .}
\end{aligned}
$$



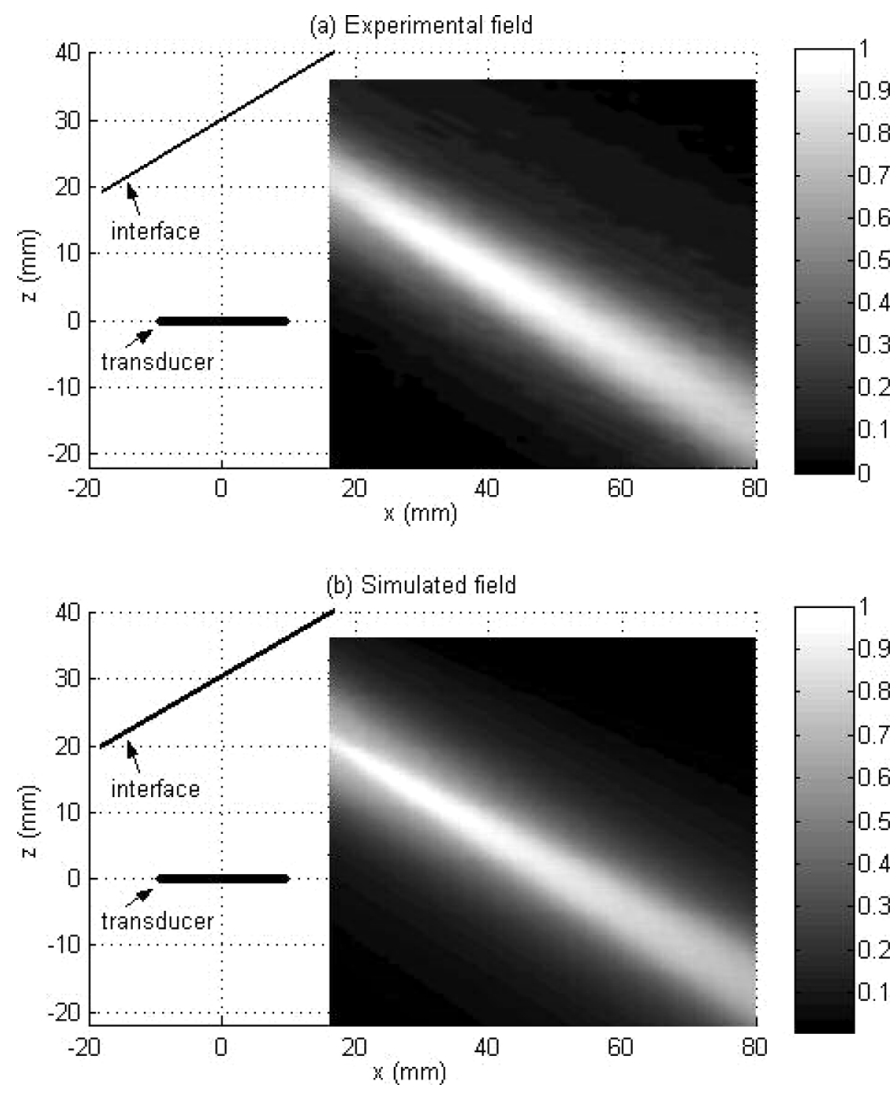

Fig. 13. The experimental (a) and simulated (b) reflected fields for $\alpha=30^{\circ}$ for water-acrylic interface.

If the function $A\left(\vec{r}_{a}\right)$ is excluded here, considering that the aperture is a uniform piston (i.e., $A\left(\vec{r}_{a}\right)=1$ ), the square-bracketed expression is exactly equal to (6), defining the impulse response $h_{a}\left(\vec{r}_{i}, t\right)$ immediately after the interface. Then, we have:

$$
p^{R / T}\left(\vec{r}_{i}, t\right)=\rho_{1} \frac{\partial}{\partial t} \int_{-\infty}^{\infty} v(\tau) h_{a}\left(\vec{r}_{i}, t-\tau\right) d \tau .
$$

If now the boundary conditions of the interface have a very significant influence to the reflected and transmitted pressure fields, we can consider a generalized expression for three basic types of baffle using an obliquity factor $\beta\left(\vec{r}_{i P}, \vec{n}\right)$ in $(3)$ :

$$
p\left(\vec{r}_{p}, t\right)=\frac{1}{2 \pi c_{\mathrm{M}}} \int_{S_{i}} \frac{\beta\left(\vec{r}_{i P}, \vec{n}\right)}{r_{i P}} \frac{\partial}{\partial t} p^{R / T}\left(\vec{r}_{i}, t-\frac{r_{i P}}{c_{\mathrm{M}}}\right) d S_{i} .
$$

In particular, the obliquity factors for rigid, soft, and free-field boundary conditions analyzed by Delannoy et al. [25] are:

$$
\beta\left(\vec{r}_{i P}, \vec{n}\right)= \begin{cases}1, & \text { rigid baffle, } \\ \left|\cos \left(\vec{r}_{i P}, \vec{n}\right)\right|, & \text { soft baffle, } \\ \left(1+\left|\cos \left(\vec{r}_{i P}, \vec{n}\right)\right|\right) / 2, & \text { free field, }\end{cases}
$$

where the modulus of the cosine guarantees its validity for the reflection and transmission cases. The generalized

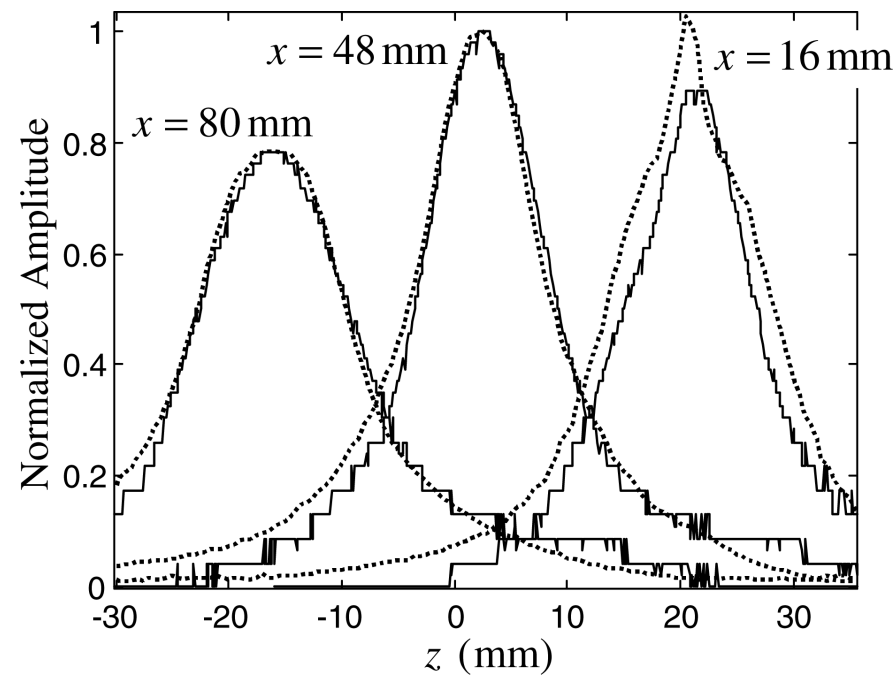

Fig. 14. Comparison of the experimental (solid line) and the simulated (dotted line) beam profiles of the reflected field from the wateracrylic interface with $\alpha=30^{\circ}$ at $x=16,48$, and $80 \mathrm{~mm}$.

expression is valid for a planar source, surrounded by an infinite baffle. However, it can approximate the radiation of a curved source under the conditions described in Section II.

Now we introduce (11) into (12) and invert the order of integration, the result is:

$$
\begin{aligned}
& p\left(\vec{r}_{P}, t\right)=\rho_{1} \frac{\partial}{\partial t} \int_{-\infty}^{+\infty} v(\tau) \\
& {\left[\frac{1}{2 \pi c_{\mathrm{M}}} \int_{S_{i}} \frac{\beta\left(\vec{r}_{i P}, \vec{n}\right)}{r_{i P}} \frac{\partial}{\partial t} h_{a}\left(\vec{r}_{i}, t-\frac{r_{i P}}{c_{\mathrm{M}}}-\tau\right) d S_{i}\right] d \tau,}
\end{aligned}
$$

where the square-bracketed expression is exactly equal to (5), defining the impulse response $h\left(\vec{r}_{P}, t\right)$ at the field point $P$, considering a soft baffle. Then, we have:

$$
p\left(\vec{r}_{P}, t\right)=\rho_{1} \frac{\partial}{\partial t} \int_{-\infty}^{\infty} v(\tau) h\left(\vec{r}_{P}, t-\tau\right) d \tau .
$$

As expected, (15) is just the convolution of the excitation signal with the impulse response at the point $P$, which is written as (4).

\section{APPENDiX B}

\section{Discrete Representation Method}

The approach proposed by Piwakowski and Delannoy [13] to calculate the field radiated from an arbitrary aperture can be described for the case of a planar aperture excited uniformly by a Dirac delta normal-component velocity pulse $v_{n}\left(\vec{r}_{a}, t\right)=\delta(t)$ and embedded in an infinite rigid baffle (Fig. 1). If the surface of the aperture $S_{a}$ is discretized by means of $N$ elements of area equals to 
(a)

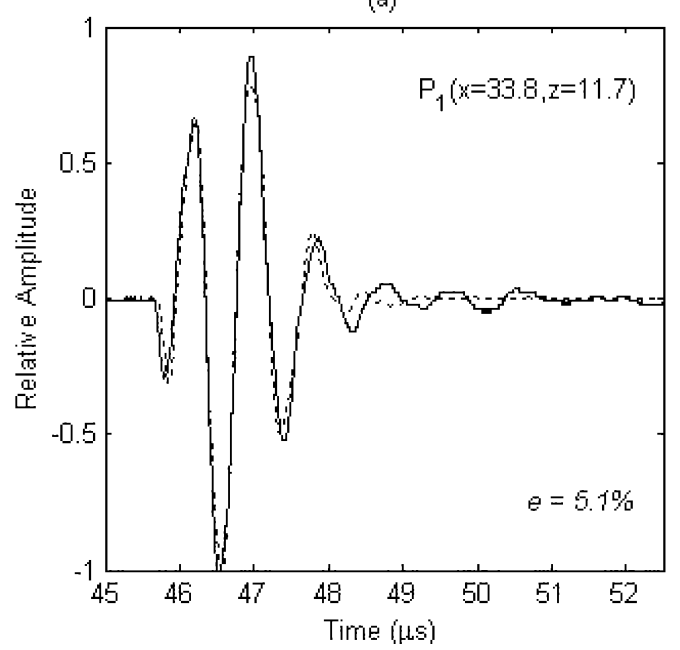

(b)

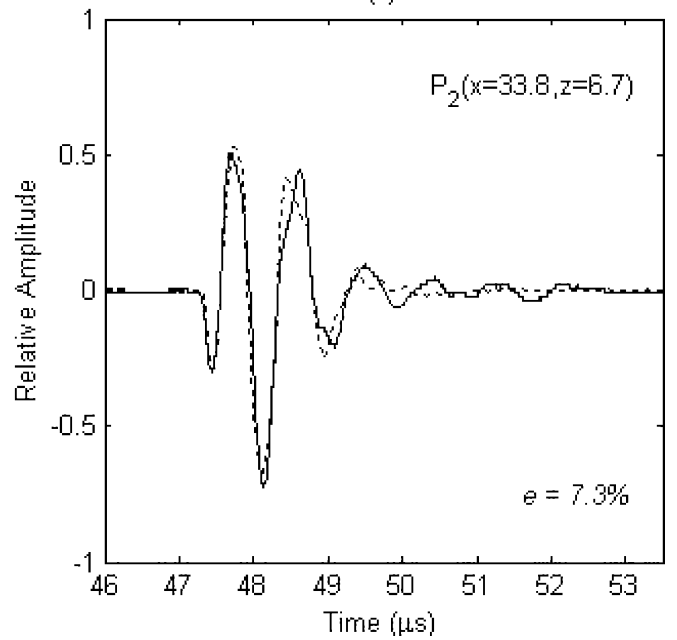

Fig. 15. Comparison of the measured (solid line) and the simulated (dotted line) pressure responses of the reflected field from the water-acrylic interface described in Fig. 13.
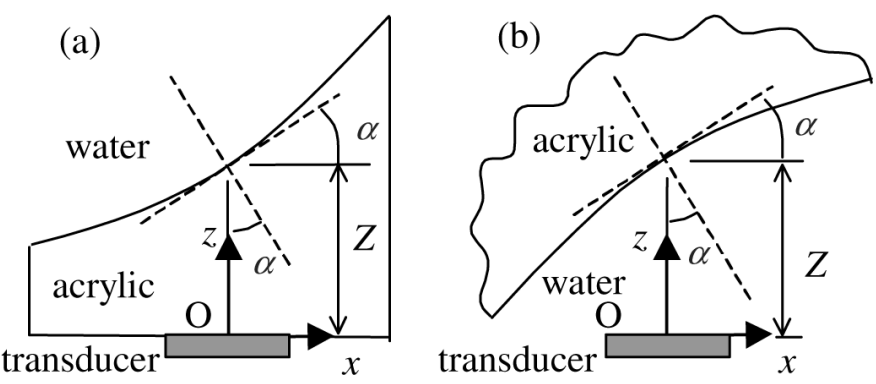

Fig. 16. The geometry of the cylindrical concave interfaces used in the transmitted field (a) and the reflected field (b). (a) Experimental field

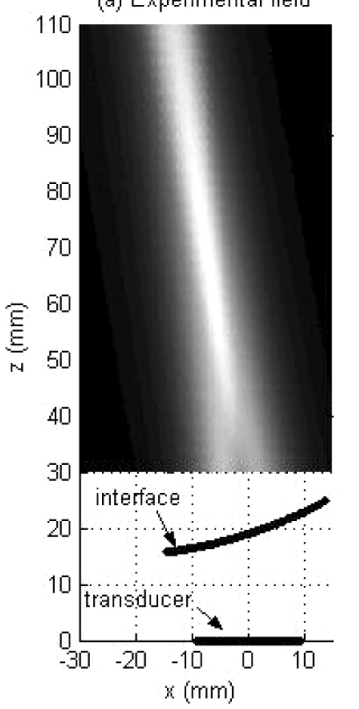

(b) Simulated field

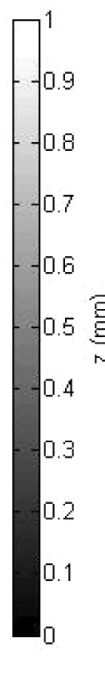

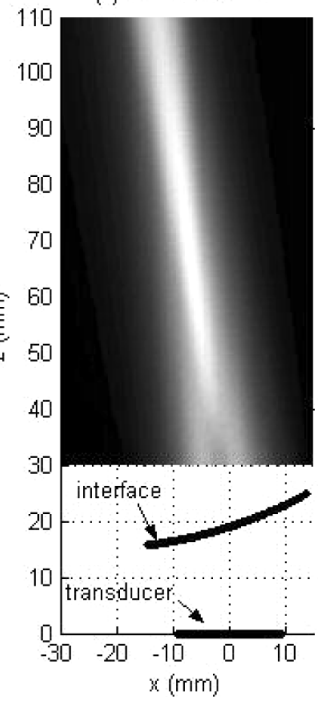

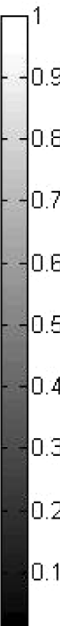

Fig. 17. The experimental (a) and the simulated (b) transmitted fields through a cylindrical concave acrylic-water interface with $83 \mathrm{~mm}$ curvature radius.

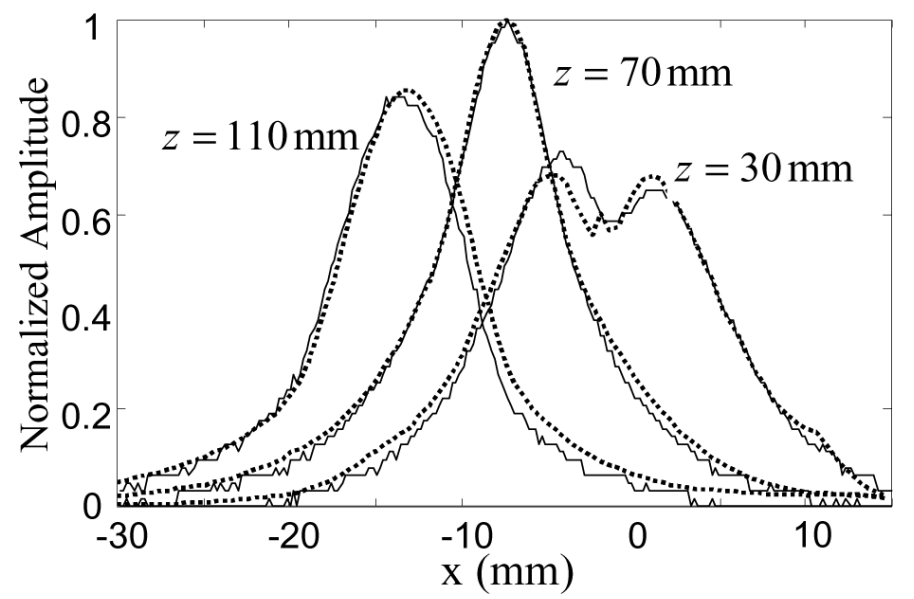

Fig. 18. Comparison of the experimental (solid line) and the simulated (dotted line) beam profiles of the transmitted field through the cylindrical concave acrylic-water interface at $z=30,70$, and $110 \mathrm{~mm}$.

$\Delta S_{j}=\Delta x_{j} \Delta y_{j}, j=1,2 \ldots N$, the Rayleigh integral for the velocity potential impulse response becomes the sum:

$$
h_{d i s c r}(\vec{r}, t)=\sum_{j=1}^{N} \frac{\delta\left(t-r_{j} / c\right)}{2 \pi r_{j}} \Delta S_{j},
$$

where $h_{d i s c r}$ is a discrete representation of the impulse response in each time $t_{j}=r_{j} / c$, and $r_{j}$ is the distance from the elementary area $\Delta S_{j}$ to the observation point at $\vec{r}$. From (16), the amplitude $a_{j}$ that represents the velocity potential impulse response generated by each elementary area, can be written as:

$$
a_{j}=\frac{\Delta S_{j}}{2 \pi r_{j}}, \quad \text { for } \quad t_{j}=\frac{r_{j}}{c} .
$$

Thus, by taking the average of all amplitudes $a_{j}$ that arrived at the observation point into the averaging window 

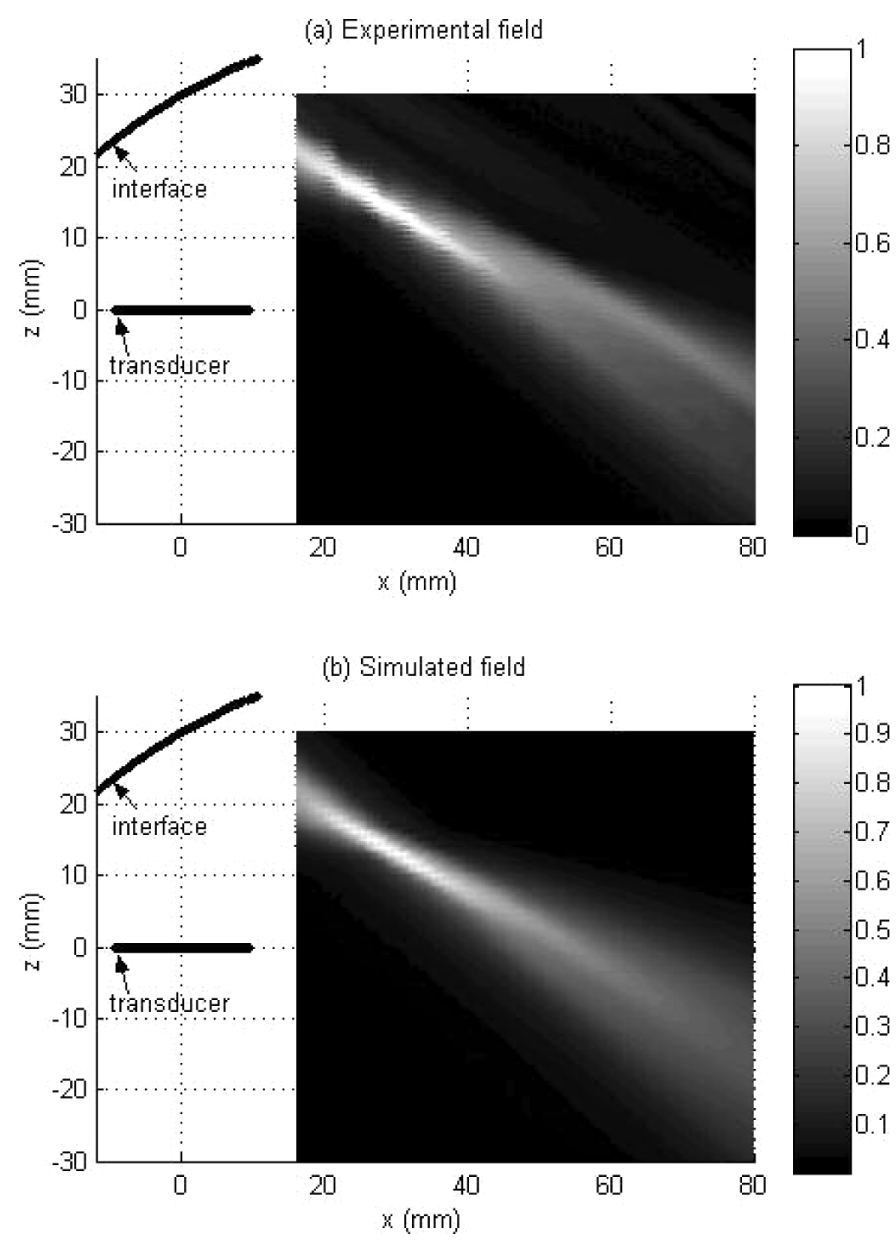

Fig. 19. The experimental (a) and the simulated (b) reflected fields from a cylindrical concave water-acrylic interface with $83 \mathrm{~mm}$ curvature radius.

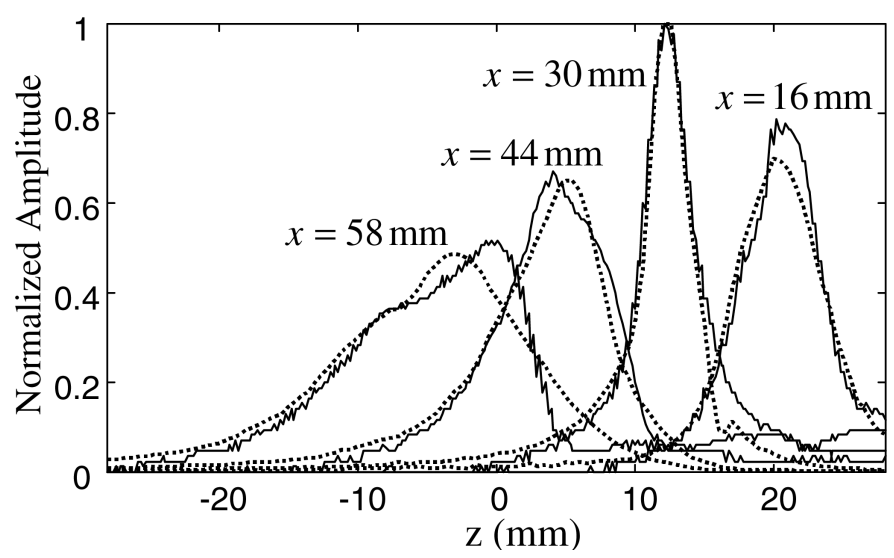

Fig. 20. Comparison of the experimental (solid line) and the simulated (dotted line) beam profiles of the reflected field from the cylindrical concave water-acrylic interface.
$\left[t_{s}-\Delta t / 2, t_{s}+\Delta t / 2\right]$, the time-averaged discrete impulse response taken at the instant $t_{s}$ is obtained:

$$
\bar{h}_{\text {discr }}\left(\vec{r}, t_{S}\right)=\frac{1}{\Delta t} \sum_{j} a_{j}, \text { for } t_{S}-\Delta t / 2<t_{j}<t_{S}+\Delta t / 2,
$$

where $\Delta t$ is the time increment used to calculate the velocity potential impulse response. It is demonstrated that $\bar{h}_{\text {discr }}(\vec{r}, t)$ tends to the analytical solution $h(\vec{r}, t)$ for the frequency bandwidth of the excitation signal $f<f_{\max }$, where $f_{\max } \ll 1 / \Delta t$, provided that the dimensions of the elementary areas tend toward zero [14].

\section{ACKNOWLEDGMENT}

The authors would like to thank the National Council for Scientific and Technological Development (CNPq), Brazil, for the postdoctoral scholarship to Flávio Buiochi in the development of this work and to the Comunidad Autónoma de Madrid (CAM) and Comisión Europea (CE) for the postdoctoral grant to Oscar Martínez. The supports from Comisión Interministerial de Ciencia y Tecnología (CICYT) DPI2001-2156-C02-02, DPI-2001-2043 and Advanced Array Technologies for Optimised Maintenance and Inspection in Critical Applications (AMICA) GRD1-CT2000-00322 EU projects are also acknowledged.

\section{REFERENCES}

[1] P. R. Stepanishen, "Transient radiation from piston in an infinite planar baffle," J. Acoust. Soc. Amer., vol. 49, pp. 1629-1638, 1971.

[2] P. Calmon and O. Roy, "Simulation of UT examinations: Modeling of the beam-defect interaction," in Review of Progress in QNDE. vol. 13, D. O. Thompson and D. E. Chimenti, Eds. New York: Plenum, 1994, pp. 101-108.

[3] P. Calmon, O. Roy, and P. Benoist, "Simulation of ultrasonic examinations," in Proc. IEEE Ultrason. Symp., Nov. 1-4, 1994, pp. $1265-1269$.

[4] M. El Amrani, P. Calmon, O. Roy, D. Royer, and O. Casula, "The ultrasonic field of focused transducers through a liquidsolid interface," in Review of Progress in QNDE. vol. 14, D. O. Thompson and D. E. Chimenti, Eds. New York: Plenum, 1995, pp. $1075-1082$.

[5] T. P. Lerch, L. W. Schmerr, and A. Sedov, "The paraxial approximation for radiation of a planar ultrasonic transducer at oblique incidence through an interface," in Review of Progress in QNDE. vol. 14, D. O. Thompson and D. E. Chimenti, Eds. New York: Plenum, 1995, pp. 1067-1074.

[6] L. Butin, A. Lhémery, and P. Calmon, "A model for predicting effects of surface wave propagation on the echo response from planar cracks," Ultrasonics, vol. 36, pp. 133-140, 1998.

[7] T. D. Mast, V. W. Sparrow, and R. Waag, "Simulation of ultrasonic pulse propagation through the abdominal wall," J. Acoust. Soc. Amer., vol. 102, pp. 1177-1190, 1997.

[8] P. P. Delsanto, N. K. Batra, R. B. Mignogna, and M. Scalerandi, "Parallel processing simulations of the propagation of ultrasonic waves through material interfaces," in Proc. IEEE Ultrason. Symp., Oct. 5-8, 1998, pp. 1129-1138.

[9] A. P. Berkhoff, P. M. van den Berg, and J. M. Thijssen, "Iterative calculation of reflected and transmitted acoustic waves at a rough interface," IEEE Trans. Ultrason., Ferroelect., Freq. Contr., vol. 42, no. 4, pp. 663-671, 1995.

[10] L. A. F. Ledoux, A. P. Berkhoff, and J. M. Thijssen, "Ultrasonic wave propagation through aberrating layers: Experimental 
verification of the conjugate gradient Rayleigh method," IEEE Trans. Ultrason., Ferroelect., Freq. Contr., vol. 43, no. 1, pp. 158-166, 1996.

[11] L. Odegaard, S. Holm, F. Teigen, and T. Kleveland, "Acoustic field simulation for arbitrarily shaped transducers in a stratified medium," in Proc. IEEE Ultrason. Symp., Nov. 1-4, 1994, pp. $1535-1538$.

[12] L. Odegaard, E. Halvorsen, B. Ystad, H. G. Torp, and B. Angelsen, "Delay and amplitude focusing through the body wall: A simulation study," in Proc. IEEE Ultrason. Symp., Nov. 36, 1996, pp. 1411-1414.

[13] B. Piwakowski and B. Delannoy, "Method for computing spatial pulse response-time domain approach," J. Acoust. Soc. Amer., vol. 86, pp. 2422-2432, 1989.

[14] B. Piwakowski and K. Sbai, "A new approach to calculate the field radiated from arbitrarily structured transducer arrays," IEEE Trans. Ultrason., Ferroelect., Freq. Contr., vol. 46, no. 2, pp. 422-439, 1999.

[15] F. Buiochi, O. Martínez, L. Gómez-Ullate, and F. Montero de Espinosa, "A computational method to predict the reflected and transmitted ultrasonic fields with interfaces of complex geometry," in Proc. IEEE Ultrason. Symp., Oct. 8-11, 2002, pp. 537540.

[16] J. C. Lockwood and J. G. Willette, "High-speed method for computing the exact solution for the pressure variations in the nearfield of a baffled piston," J. Acoust. Soc. Amer., vol. 53, pp. 735-741, 1973.

[17] D. E. Robinson, S. Lees, and L. Bess, "Near field transient radiation patterns for circular pistons," IEEE Trans. Acoust. Speech Signal Processing, vol. ASSP-22, no. 6, pp. 395-403, 1974.

[18] M. A. Fink and J. F. Cardoso, "Diffraction effects in pulse-echo measurement," IEEE Trans. Sonics Ultrason., vol. 31, no. 4, pp. 313-329, 1984.

[19] J. P. Weight, "Ultrasonic beam structures in fluid media," $J$. Acoust. Soc. Amer., vol. 76, pp. 1184-1191, 1984.

[20] D. A. Hutchins and G. Hayward, "Radiated fields of ultrasonic transducers," in Physical Acoustics: Principles and Methods. vol. XIX, R. N. Thurston and D. Pierce, Eds. New York: Academic, 1990, pp. 1-80.

[21] J. W. Goodman, Introduction to Fourier Optics. 2nd ed. New York: McGraw-Hill, 1996, pp. 32-62.

[22] H. T. O'Neil, "Theory of focusing radiators," J. Acoust. Soc. Amer., vol. 21, pp. 516-526, 1949.

[23] A. Freedman, "Sound field of plane or gently curved pulsed radiators," J. Acoust. Soc. Amer., vol. 48, pp. 221-227, 1969.

[24] A. Penttinen and M. Luukkala, "The impulse response and pressure nearfield of a curved ultrasonic radiator," J. Phys. D: Appl. Phys., vol. 9, pp. 1547-1557, 1976.

[25] B. Delannoy, H. Lasota, C. Bruneel, R. Torguet, and E. Bridoux, "The infinite planar baffles problem in acoustic radiation and its experimental verification," J. Appl. Phys., vol. 50, pp. 5189$5195,1979$.

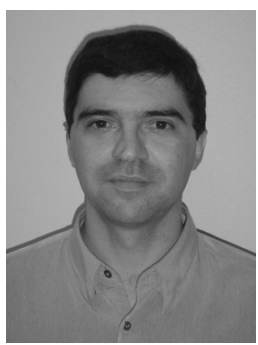

Flávio Buiochi received the mechanical engineering degree, and the master and doctor degrees in mechanical engineering from the Escola Politécnica da Universidade de São Paulo, Brazil, in 1990, 1994, and 2000, respectively. He started his teaching career at the Universidade de São Paulo Departament of Mechatronics, São Paulo, Brazil, in 1992, where he is currently an assistant professor. From September 2001 to March 2003, he was a postdoctoral Fellow in both Instituto de Acústica and Instituto de Automática Industrial of the Consejo Superior de Investigaciones Científicas of Spain, Madrid, Spain.
His research interests include applications of ultrasonic transducers in nondestructive testing and characterization of liquids by ultrasound.

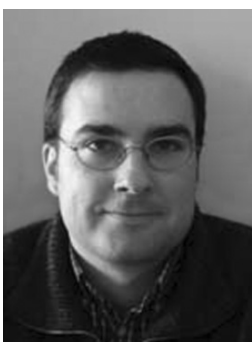

Oscar Martínez was born in Valencia, Spain. He received the telecommunication engineer degree in 1995 from the Polytechnic University of Valencia, and his Ph.D. degree from the Polytechnic University of Madrid in 2000 .

Since 1995 he has been at the Instituto de Automatica Industrial of the Spanish Council Scientific Research (CSIC), Madrid, Spain. He is involved with ultrasonic imaging, ultrasonic arrays, digital signal processing, and real-time architectures.

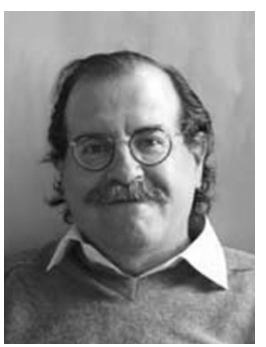

Luis Gómez-Ullate was born in Ciudad Real, Spain. He received his master's degree in industrial engineering from the Universidad Pontifica de Comillas, Madrid, Spain, in 1968, and his Ph.D. degree from the Polytechnic University of Madrid in 1990.

Since 1972 he has been at the Instituto de Automatica Industrial of the Spanish Council Scientific Research (CSIC), Madrid, Spain, where he has worked in different scientific areas relative to automatics and signal processing.

Since 1985, his main areas of interest are ultrasonic imaging, array transducers, modeling, real-time systems, signal processing, and diverse techniques for nondestructive testing applications.

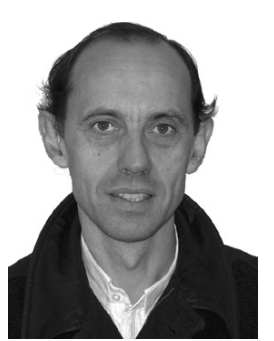

Francisco Montero de Espinosa (M'91) was born in Madrid, Spain, in 1955. He received the M.S. and Ph.D. degrees in physics from the Universidad Complutense of Madrid. $\mathrm{He}$ received a grant at the Instituto de Acústica, Spanish Council Scientific Research (CSIC), Madrid, Spain, as postgraduate student, becoming a member of the scientific staff in 1986. At present he is research professor.

His main areas of interest are ultrasonic transducers in general, piezoelectricity, acoustic field, piezocomposite materials, echography and NDT, and physical acoustics. Current research projects deal with air-coupling piezoelectric array transducers, cMUT, and food testing by ultrasound in lab and industry. 